\title{
NEW PERSPECTIVES ON PROTECTION AGAINST POSSIBLE USE OF BIOLOGICAL WEAPONS
}

\author{
Sergej Cvetovski, PhD \\ Faculty of Philosophy - Institute of Security, defense and peace \\ E-mail: sergej@fzf.ukim.edu.mk \\ Vanco Kenkov, PhD \\ Faculty of Philosophy - Institute of Security, defense and peace \\ E-mail: vancok@fzf.ukim.edu.mk
}

\begin{abstract}
Unlike nuclear and chemical weapons, biological weapons are means of mass destruction which is easily accessible. Open use of biological weapons in a military conflict it is very unlikely, but it is more realistic its application as a means of conducting subversive war and terrorism. Protection against the effects of biological weapons comes down primarily to the application of measures that are used in the prevention and treatment of contagious diseases. The authors of this paper neither diminished the role in the prevention of biological attacks of the appropriate security and intelligence services. The contents of this paper highlight issues of: the challenge of recognizing biological attack, the methods of isolation and detection of harmful microorganisms by classical microbiological methods and their weakness, the agents altered resistance to existing antibiotic and (im) possibilities for effective protection. The authors also presents the prevailing scientific conflicting views on the horror of the possible use of genetically modified organisms and opposing soothing views indicating that such opportunities are premature. The analysis of capabilities for vaccination, as a specific defensive measure of protection in case of biological attack, suggests that capacities are fairly limited. The methods of biological decontamination show that this is a delicate procedure.
\end{abstract}




\section{Seccurity}

Key words: biological weapons, biological agents, attacks, infectious disease, protection.

\section{Introduction}

Biological weapons is constituted of pathogenic organisms or their toxins which are used in a biological attack. Often referred to as bio-weapons or agents of biological threat or just bio-agents. They are living organisms or replicated entities (such as viruses which generally are not considered alive) that reproduce or replicate in their hosts casualties. Insects in entomologic warfare is also considered a form of biological weapons. The use of biological weapons by terrorist groups or individuals sects is referred to as biological terrorism (bioterrorism) and it is deliberate release and dissemination or biological agents. According to the United States Centers for Disease Control and Prevention, bioterrorism is the deliberate release of viruses, bacteria, toxins or other harmful agents causing disease or death to humans, animals or plants. Also taken into consideration and agents that do not harm people directly, but which disrupt the economy.

Unlike nuclear and chemical weapons, biological weapons are a means of mass destruction which can be obtained even by the poorest nations or certain groups. Biological weapons allows potential of causing the level of destruction and loss of life far more than conventional, chemical or even nuclear weapons, compared with its mass and cost of production and storage. Biological agents can be developed, procured, stored and implemented by states or non-state actors. In the latter case, or when the state uses it secretly can also be considered as bioterrorist Act. (Wheelies Mark et all. 2006).

There is question which are who these organisms or their toxins which could be probably used as a biological weapon ?! In case of bacteria first of all it would be all bacteria causing anthrax, plague and tularaemia and in case of viruses it is all of them causing smallpox, venezuelan equine encephalitis, Ebola, Lassa and Marburg fever. Rikecijata- Coxiella burneti, causing Q- fever can also be used as a biological weapon as well as botulin toxin, staphylococcal enterotoxin B and ricin toxint. Toxins are often referred to as midspektar agents that differ from others bioweapon by not reproducing in their hosts and by a shorter incubation period. Here, we should also mention the 
entomological warfare (EW), a type of biological warfare that uses insects to attack the enemy. This concept has existed for centuries and researching and development has continued to the modern era. Entomological warfare uses insect for direct attack or as vector to transfer biological agent such is plague. Basically, EW exists in three variants. The first type of EW involves infecting insects with a certain pathogen insects and distributing them over the target areas. Then insects act as vector, infecting each human or animal that would bite. Another type of EW is a direct attack of insects against crops; where the insect may not be infected with a pathogen, but serves as direct threat to agriculture. The last way uses uninfected insects, such as bees, wasps, etc., as a direct attack to enemy. (Lockwood 2008, 9-26)

The goal of action of biological agents can be humans, animals and agricultural products. Under the former program of the US biological warfare, the anti-human bioagents are divided into Deadly agents (like anthrax, French tularemia, botulinum, etc.), and disabling agents (such as brucellosis Suisse, Kohili, Venezuelan equine encephalitis virus, staphylococcus enterotoxin $B$, etc.). Biological warfare as specific goal can have plants in order of devastating harvest or defoliating the vegetation leaves. The attack on animals is another area of biological warfare intended to eliminate animal resources used for transportation and food.

\section{Use of biological weapons}

Biological weapon can be used in different ways in order to gain strategic or tactical advantage over the enemy, or as threat of use or as actual use. As some part of the chemical weapons also biological weapons can be used as a weapon for enemy deflection. Hence, biological agents may be useful as a strategic deterrent in addition to their usefulness as offensive weapons on the battlefield. The agents can be fatal or nonfatal and can aim at an individual, group of people or an entire population. As tactical weapons in military use, a significant problem with biological weapons (BW) used in the attack is that you have to spend days to become effective and by that it may be impossible to quickly stop the opponent's force. Some biological agents (such as smallpox or pneumonic plague) have ability to transfer from one person to another through respiratory aerosol particles. 


\section{Seccurity}

Today is unlikely that a State would have decided openly to use the biological weapons in a military conflict, bearing in mind the uncertainty of achieving desired effects and the real possibility of losing control over them. This is undesirable feature, because with this technique, agents can be transmitted to unintended population, including neutral and own forces. In that sense, BW is less concern that might come from certain criminal or terrorist organizations than the serious concern of the military and civilian population of any nation. Hence, more realistic is its application as a means of conducting subversive war, and to encourage and controlling political crises. Because certain types of biological weapons can be produced even in the simplest microbiological laboratories, the biological weapons is quite

Historically, biological warfare has been applied since the ancient times. In the 6th century BC Assyrians had contaminated enemy wells with a fungus which caused delirium at their opponents. In the new era in 1346, the Mongols, has catapulted the bodies of their comrades who died from the plague inside siege walls during conquering the Crimea. Historians also debate that the army of Britain has used smallpox against Native Americans. With advances in bacteriology since 1900 was provided a higher level of sophisticated techniques for possible use of bio-agents in the war. Sabotage in the form of anthrax was applied by Imperial Germany during World War I, but with insignificant results. During World War II, the Great Britain established Porton Down BW program and develop its BW by introducing the industrial manufacture of a variety of deadly pathogens, although offensive has never used that weapons. In this period, other nations like the US, France and Japan has also started a BW program (Garrett, 2003, 341). The most notorious program in that time was led by Unit 731 of the Imperial Japanese army located in Manchuria. This unit was conducting a fatal human experiments with prisoners and produced BW for combat use. Although the Japanese lacked the technological sophistication of the programs of their opponents, however, they exceeded them with the widespread use of BW and with indiscriminate brutality. US and Britain during World War II discovered plant growth regulators (ie herbicides) and started warlike herbicide program that will be used later in counterinsurgency operations in Malaysia and Vietnam.

Simulated attacks with biological weapons have shown that even the developed countries are quite vulnerable and that biological weapons can easily be used, for 


\section{Seccurity}

example, through the ventilation systems in underground railways. ${ }^{177}$ The effectiveness of biological products such as biological weapons illustrates the fact that some biological toxins (such botulinum toxin, shiga toxin and ricin toxin) are more deadly than chemical toxins that are anticipated to be chemical weapons (such as Soman, Sarin, VX).

Protection from use of biological weapons primarily comprises applying of measures that are used in the prevention and eradication of infectious diseases. Also, it should not be denied any significant role in the prevention of biological attacks that have appropriate security and intelligence services, which are involved in data collection about intentions of potential adversaries, possible locations and time of use of biological weapons, and the type of biological agent. In reality, their actions in practice is rarely successful, so most used measures of this protection would be implemented after exposure to biological weapons occur. Foremost such measures are: a) identifying the biological attack, b) detection and identification of biological agents, c) taking care of exposure persons, d) biological decontamination.

\section{Recognition of biological attack}

The main problem here is determining whether a possible situation is a matter of natural occurred disease or of biological attack. This can sometimes be a great challenge, because the one who uses biological agents as weapons might use all epidemiological and environmental circumstances of a particular territory to impede or prevent the recognition of a biological attack. Even in a situation where those circumstances are not used to cover up the eventual attack, the element of deliberate use is hardly recognizable.

A robust surveillance system which includes medical doctors and veterinarians could detect biological attack early in the epidemic, allowing prophylaxis of the disease in the majority of people (and / or animals) that are exposed but are not yet ill. If there

\footnotetext{
177 In March 1995 the Aum Shinrikyo sect used chemical poison sarin, during there were 11 deaths and at least 5500 infected people. Later, in the premises of this sect was also found biological weapons. It is estimated that the death toll would have been much larger if this sect in Tokyo subway attack instead sarin would used botulinum toxin and anthrax.
} 
is doubt that it is an issue of biological attack, it is necessary to conduct field epidemiological investigation in order of relevant data collection, which will be in function of acceptance or rejection of earlier doubt. To possible biological attack indicate certain specificities as (Treadwell, 2003, 93-94):

- Sudden and unexpected occurrence of frequent illness and / or death which is not related to previous epidemiological situation,

- Simultaneous infection of patients with two or more causers;

- Unusual geographic occurrence of infectious diseases (such as mass disease in cities with Q -fever, tularemia, or anthrax, and occurrence of rare or exotic diseases - Lassa, Ebola or Marburg haemorrhagic fever in our geographic area);

- An outbreak of seasonal diseases at time when usually are not register;

- Unique reason for a disease caused by an unusual agent, with lack of epidemiological explanation;

- Frequent illness and deaths of animals in relation to patients with similar symptoms;

- Existing evidence of contamination of air, water, food (through the remains of bombs, equipment and means of water and food contamination, report data, etc.);

- The uncommon, rare, genetically modified strain of the agent;

- Unregistered disease in people who are not exposed to common ventilation system when the disease is observed in individuals who have a common ventilation system;

- A rare disease that is uncommon to a particular age group or population where occurs;

- Increased number of applicants for medical treatment at the same time and abundance of cases of unexplained illness and death.

When suspected biological attack it is necessary as soon as possible to determine the way that has led to emergence of the disease in order of establishing effective measures of suppression. For easier and quicker orientation, based on the leading clinical symptoms and signs of disease, should set clinical-epidemiological syndrome diagnosis and registered disease be classified as any of following syndromes: respiratory syndrome tract- where the likely route of transmission of infection is air; syndrome of gastrointestinal tract - where transmission route is water or food; syndrome of central nervous system - where the transmission paths is air and vectors 


\section{Seccurity}

(which traditionally in medicine organisms which do not cause disease themselves, but the ones who spread infection conveying pathogens from one host to another) ${ }^{178}$; Syndrome of change of the skin and visible mucous membranes - with the possible route of transmission as: air, vectors, contact, and Hemorrhagic syndrome-with possible transmission by air and vectors.

\section{Detection and identification of biological agents}

The armies of some countries have developed systems and instruments for the detection of the biological weapons immediately after its use, such as the US Army with "LIDAR" system for detection of infectious aerosols in the air. These systems have proved useless because have detected all the particles present in the air, without differentiating infectious from not infectious one, usually leading to a number of false alarms (Čobeljić M. 2001: 206). The growing threat of biological warfare agents and bioterrorism has also led to the development of special field instruments for analysis and identification on the site of the suspicious material which is found. One such technology that has been developed by researchers (at Lawrence Livermore national laboratory-LLNL), uses the method of "sandwich immunoassay" in which fluorescent labeled antibodies aimed at specific pathogens are attached to silver or gold nanowires. Researchers at Ben-Gurion University in Israel have developed a different device called a BioPen, which can detect known biological agents in less than 20 minutes by using an adaptation of ELISA- the similar widely used immunological technique, which in this case includes an optical fiber. In the Netherlands, the company TNO has designed the equipment for identifying specific particles in aerosols (BiosparQ).

(Richmond and McKinney 1999). Collection of material for laboratory testing is necessary to be done by staffs that are trained to take samples, to transport those samples and to protect themselves. In assessing the capacity of microbiological laboratories working on detection and identification of biological agents, in addition to diagnostic capability, the decisive influence has the existence of appropriate conditions for protection of personnel and environment, i.e. appropriate level of biological protection -BSL which ranges from BSL1 to BSL4. (Richmond and McKinney 1999).

${ }^{178}$ For example, the species of mosquito, who serves as vectors for a deadly disease Malaria. 
The materials in which can be detected biological agents related the risk of occurrence and spread of infectious diseases can be sorted into three categories: low contaminating and uncontaminating materials; moderately contaminating materials; and highly contaminating materials. The first category (low or uncontaminating materials) encompasses the biological material (samples of tissue, blood, urine, feces, etc.) of those suffering from some infectious diseases transmitted by biological vectors (as typhus, malaria, yellow fever, Lyme disease, tick-borne meningoencephalitis) or those poisoned with toxins from microorganisms such as botulinum toxin ricin toxin, aflatoxin. In these cases there is no transmitting interhuman disease. Moderate contaminating material is water, food or biological material where can be found samples of typhoid, bacillary dysentery, tularemia, brucellosis, glanders, anthrax; where there is possibility for interpersonal transmission. Highly contaminating material is one of the easy-to-create aerosols, such as dust or spray in witch can be found causers of anthrax or plague, as well as biological materials of those suffering from smallpox or hemorrhagic fever (Marburg, Ebola, Lassa). This material commonly, is suitable to interpersonal spread of the disease. Depending on the category of contaminating material, the adequate level of biological protection it is required.

For the isolation and detection of microorganisms the most reliable are classic microbiological methods, but their main weakness is the long time period required to obtain the final results: 2-4 days for bacteria, 3-10 days for viruses, and several weeks for lichens. Because is essential as soon as possible to detect which agent concerned, rapid methods of detection and identification are used, as (immuno) luminous microscopy detection of antigens of microorganisms with immunoenzyme tests (ELISA), and methods of molecular genetics that multiplies and detect genetic material of microorganisms (PCR and DNA hybridization).

In theory, the new approaches in biotechnology, such as synthetic biology can be used to design new types of warfare agents. Particular attention should be paid to future experiments because of concern: of a demonstration that vaccines can become inefficient; that will contribute to resistance to therapeutic antibiotics or useful antivirus agents; of possible strengthening of the virulence of the pathogen or generating a non-pathogenic contamination; of possible increased transmissibility of the pathogen; of modification of range of the pathogen host; of enabling avoidance of diagnosis tools; and possibility of biological agent or toxin to be used as a weapon (Kelle 2009). 
New opportunities for the development of biological weapons such as recombinant DNA technology are the greatest horror for those working on developing methods to detect and to combat the consequences of the use of biological weapons. The danger stems from the fact that by genetic manipulations can be obtained microorganisms that are more virulent than their natural relatives. The fear that such a development of biological weapons could happen is more justified because a decoding of the complete genome of certain pathogens have become common approach in research and because the techniques of manipulation of the genes are being more and more developed. Many companies have conducted research in terms of targeted molecular evolution, a term that implies accelerated evolution aimed at wanted direction, so that in some microorganisms are introduced genetic variation and then artificial selection is applied. These methods are currently too complicated to be applied by bioterrorists but not unreachable for institutions in countries that want to develop biological weapons. A terrifying case suggests that in this way an Escherichia coli kind was obtained with gene of a resistance that is transferred from staphylococcus so that new species become 32000 times more resistant to third generation cephalosporin antibiotic than the wild species. Due to a diligence of possible abuse this kind bacterium was immediately destroyed (Dennis 2001).

Biological weapons experts say there is still no possibility for preparation of new agents, but it is quite certain that the current ones can be made more virulent, resistant to a greater number of antibiotics or altered to such an extent that they cannot be detected by conventional diagnostic procedures. The simplest way to get the improved biological weapons is making the existing agent more resistant to existing antibiotics. The genes for antibiotic resistance are found in the genome of the microorganism, usually as part of the extra chromosomal genetic elements - plasmids that have the ability to transfer and replicate independent, and often contain other virulence genes. Laboratories dealing with molecular biology have no problem transferring selected genes from one bacterium to another ${ }^{179}$. Recently appeared the system CRISPR / CAS as a promising technique for correcting genes. While other

\footnotetext{
${ }^{179}$ According to Alastair Hay, an expert on biological weapons of Leeds (UK), in the institutions of Biopreparat (Russia and Kazakhstan) which concerned the development of biological weapons, was developed Yersinia pestis, which is resistant to 16 different antibiotics.
} 


\section{Securianues}

methods require months or years for the sequencing of genes, CRISPR reduces that time up to weeks (Basulto. 2015).

Although most experts in the field of biological weapons, thinks that its premature fear of germs which are created by genetic manipulation and that is greater need to think of conventional biological agents, the government agencies in the United States are already engaged in developing methods for detecting diseases caused by a genetically modified microorganisms.

The basic principle of detection methods is based on knowledge of what should be looked for. Thus, RSVP (Rapid Syndrome Validation Project) is an electronic system that is connected to the Internet, which puts the probable diagnosis of the syndrome based on previously entered data on uncommon disease or unusual epidemic. Also, developed is a system which can detect the presence of unknown causer solely based to the presence of molecules or biological toxins in the investigated material. DARPA Agency (US Defense Advanced Research Projects Agency) has developed a sophisticated system of biosensors based on living tissues that respond to a wide range of known and unknown pathogens. The biosensors are three-dimensional matrices containing neurons, muscle cells, cells of the immune system and skin cells and epithelial cells surrounding the digestive system and the nasal mucosa (Dennis 2001).

\section{Protection and care for exposed diseased and healthy individuals}

Against biological warfare agents protection measures can be applied. Those measures are need to start on time (if sufficient warning is received), but definitely as soon is suspected that biological agent it used. The basic protection is done by means of personal protection, such as masks and protective clothing. Currently available military protective mask masks or HEPA is highly efficient filter mask for airborne particles used in exposure with tuberculosis- it filter out most particles of biological warfare present in the air. ${ }^{180}$ Most biological agents in the air can not penetrate the undamaged skin, and is little organisms stucked to the skin or clothing. After the attack with aerosols, the most common removal of clothes eliminates the majority of surface

\footnotetext{
${ }^{180}$ For the mask tight, it must be adjusted / stick to the face of the user.
} 
contamination. Thorough shower with soap and water removes $99.99 \%$ of those little organisms that may remain on the skin of the victim.

The following procedure that is to be applied is medical care. The care of patients, in general is conducted in the area of biological attack (healing on spot), in the health facilities where are existing conditions for adequate assistance and adequate level of biological protection. Because on the onset of the biological attack is not known what agent is run, it is needed by the medical personnel and others involved in care, to apply all available measures of medical and technical protection. The medical professionals who treat victims of biological warfare may not need to use special protective suits, but should use rubber gloves and apply additional precautions like wearing gowns and eye protection masks. If there are indications that it is highly contaminating causer, are being applied measures of strict isolation of patients, suspected of disease suffering and the staff who care for them, from the rest of the population. For isolation if there are capacities, separate rooms to be used for each patient or patients to be grouped based on the clinical syndrome, separate from other patients in different rooms. If this is not possible due to a number of cases and / or an insufficient number of hospital facilities so that patients regardless of the reason for the hospitalization must be placed in common areas, it is necessary to provide at least minimal spatial separation of each other. In the event of mass illness also is possible establishing cohorts of patients in these rooms (Judith et al., 1999, 6).

Transport and movement of patients is maximally restricted, and if their movement is still necessary, is needed to take all precautions to prevent further spread of infection ${ }^{181}$. If the available quantities of medical equipment and resources permit, it should be used for each patient individually, or possibly a group of patients with the same clinical syndrome. If for any reason that is not feasible, the potentially contaminated equipment should not be used in the care of other patients until is properly disinfected or preferably sterilized.

At beginning treatment is implemented on the basis of the established clinicalepidemiological syndrome diagnosis. If there is suspicion of a bacterial infection, antibiotics are given, with different antibiotics are given to various small groups of patients, so that as soon as possible based on "ex juvantibus" outcome to get to discovery which antibiotic is most effective. Such therapy may impede the use of

${ }^{181}$ Such as placing a mask of the patient to prevent spread of airborne infection. 
multiresistant bacteria as a biological weapon. When the final results of laboratory analysis (identification of the agent and antibiogram) are got, therapy can be corrected if needed. The victims of biological warfare may be given antibiotics orally or intravenously, even before the specified agent to be identified. As previously mentioned, the bacterial pathogens in a possible biological attack would probably be used those that causes anthrax, tularemia and plague. These agents are therefore grouped in the so-called Category A biological agents in accordance with US authors and there are some recommendations ${ }^{182}$ which antibiotics will be selected for treatment, prevention and postexposure prophylaxis of infections caused by these pathogens (Navas, 2002). For the treatment of those suffering from anthrax as first choice drugs are recommended doxycycline or quinolones for 60 days. In the treatment of tularemia, the drug of choice is streptomycin or gentamicin for 7 to 14 days. Tetracycline and chloramphenico/ have bacteriostatic activity and are effective if given at least 14 days, with more frequent relapses than when giving streptomycin. Streptomycin was the first drug of choice in the treatment of plague, but if it will be used gentamicin and tatraciklin, chloramphenicol and quinolones are an alternative choice. They are effective if used at the time (within 24 hours of onset of pulmonary plague).

Specific measure of counter-biological protection in healthy persons is a prophylaxis, which due to the urgency of its application is called emergency prophylaxis and is presented in two types: pre-exposure prophylaxis and postexposure prophylaxis. It covers the use of vaccines, specific immunoglobulins (immunoprophylaxis) of antimicrobial agents, eg. antibiotics (in chemoprophylaxis) and antitoxins. The application of antibiotics in postexposure prophylaxis, as well as in the case of treatment of patients, should start as soon as possible, and this treatment should last long enough.

As a specific measure also can be used vaccination, primarily for pre-exposure prophylaxis, although it can be also applied post exposure, which depends on the length of the incubation period. Currently, protective vaccines given as injections are available for anthrax, Q-fever, yellow fever and smallpox. Widespread immunization of nonmilitary personnel is not being recommended by any government agency. Immunoprotection against toxins of ricin and staphylococcus might be available in the near future.

\footnotetext{
${ }^{182}$ Working Group on Civilian Biodefense.
} 
It should be noted that capacities for vaccination, as a specific defensive measure of protection in case of biological attack, are rather limited. Effective protection of the armed forces (not mentioning protection of the entire population) against the full range of biological agents that a potential attacker could use is impossible, because such prophylaxis is specific, so protection can only be achieved against a certain type of pathogen. Unfortunately, inducing a protective response to vaccination may last longer than the time from exposure to onset of illness. Moreover, many vaccines require multiple doses in achieving a protective immune response, which limits their usefulness in emergency vaccination programs in providing prophylaxis in case of quick attack. In fact, not all recipients of the vaccine showed protective reaction, even after receivement of the recommended vaccination stack. People with impaired immunity are often unable achieving an effective response to vaccination and certain vaccines may be contraindicated for them. For example, a vaccine against hepatitis $B$ does not cause antibody response in approximately $10 \%$ of the vaccinees, where percentage of those not-reacting is even larger at immuno-impaired persons. (Pirofski and Casadevall 1998). Given the large number of micro-organisms and their toxins that can be used as a biological weapon, of great importance are reporting data for biological agents that are available to potential enemy in protecting its own population. But even when such data are available, however, the effective mass vaccination is a major problem. First, there should be an effective vaccine, then it should be sufficient supplies of it and as a last problem is the needed time achieving a satisfactory level of immunity after vaccination (Geissler 1986) ${ }^{183}$. Therefore, vaccines cannot be considered as first line of defense against bioterrorism for the general population, as with case with relatively small military population.

Vaccines are mainly useful for an attacker, because if has an effective vaccine, he is able to adequately protect its own armed forces and population. Thus, the attacker eliminates one of the distinguishing features of biological weapons, which limits its use

\footnotetext{
${ }^{183}$ In the US it was estimated that in case of biological attack with Eastern encephalitis virus, only half of Division (about 8,000 people) could be immunized with the vaccine reserves stored in the US Army. It would take three months to produce enough vaccine to protect the rest of the division, and even about a year to immunize an independent corps of five divisions (i.e. about 190,000 people). In addition, it would take several weeks or months, vaccinated people to achieve an adequate level of immunity.
} 
of military aspect, -it is the danger that use of biological weapons cause losses in their own ranks.

Vaccines are an essential component of defense strategies by its application in uniformed military personnel. There are licensed vaccines against anthrax, smallpox and plague. Moreover, new products like drugs in experimental stage are being applied in the Medical research centre on infectious diseases of the US military (USAMRID) for staff protection in risky laboratories against tularemia, $Q$ fever, Venezuelan equine encephalitis (VEE) and botulinum, and against diseases such as Eastern and Western equine encephalitis, Rift Valley fever and others. Ministry of Defense of US recently began a campaign for immunization against anthrax in the armed forces and is quite likely that other vaccines against bio-warfare will eventually be used in protection of the armed forces. Currently, vaccines against other biological agents and improved vaccines for agents listed above, are already in various stages of research and development. As problematic remains the use of these vaccines in the civilian context because that there nature of the threat is less defined. However, some vaccines like those against anthrax and smallpox may have applicability to post-exposure prophylaxis and handling exposed civilian populations. (Cieslak et all, 2000, 849).

\section{Defensive strategy based on passive antibodies}

As a new feature in protection against biological agents is a newly proposed defense strategy based on antibodies. The introduction of antibodies taken from immune donors in non-immune individuals is known as passive immunization. These "borrowed" bodies offer short-term protection from certain diseases. The application of this strategy, today can be supported by modern technology. Therapies based on antibodies were first used at the end of the 19th century, and so far in this area there is more than 100 years of experience with the development of therapeutic antibodies. In the past, therapies based on antibodies were dependent of immune serum which was limited in availability and taunted significant side effects when serum was obtained from animals.

Storage of reagents based on antibodies that could quickly be given the exposed population would greatly reduce the threat of many biological agents by providing a way of granting immediate immunity of susceptible persons. In cases of 
existing dangers where vaccines are available, this measure would provide additional time for immunization and would reduce risk. The development of therapies based on antibodies may reduce the attractiveness of biological warfare as a means of terror by providing antidots that would help neutralizing of the threat. The eventual aggressor could try to defeat the passive defense based on antibodies by discovering an agent which could express the antigenic changes, the proteases or antibody binding protein. However, in this race of armaments, the advantage may be in favor of defender because it is technologically easier to create effective new antibody against the modified agent than to produce pathogen or agent for enhancing the virulence. Antigenic changes by default create epitopes ${ }^{184}$. It is also possible creating antibodies that resist proteolysis by changing the sequence of amino acids to eliminate the proteolysis' location. In fact, a means of neutralizing antibodies could be created much faster than it can be developed a new biological agent. An example of the speed by which can be developed therapeutic antibodies comes from the 1905 epidemic of meningococcal meningitis in New York when Simon Flexner created effective horse antiserum in several months and used it to treat patients before the epidemic can be naturally reduced. Although this example is not applicable today, given the regulations for the development of therapies, however, it shows a dramatic example of a concept where therapies can be developed quickly. The same does not goes for new antimicrobial chemotherapy or vaccines, which often require a significantly longer time to develop.

Candidates for this potential use of passive immunization are botulinum toxin, tularemia, anthrax and plague. For most of these goals, has been conducted only animal studies, so the use of passive immunization in the possible event of applied bioweapon is still in the experimental stage. Because companies cannot ethically expose people to deadly agents, they can never be completely sure that the drug / vaccine acts against a specific danger. Experiments with people are conducted generally only for security purposes. Additionally, it is also problematic combining the results from studies with animals and the results of trials in favor of human security, implying only a high degree of probability that these vaccines will affect people. Most animal models are not as good at predicting how a man would react to certain agents and to drugs intended for agent countering.

${ }^{184}$ Epitope by definition is part of the antigen molecule to which antibodies attach themselves. 


\section{Securiagus rity}

\section{Biological Decontamination}

For biological agents, contamination is defined as the introduction of microorganisms in tissues or sterile materials, while decontamination is defined as disinfection or sterilization of contaminated parts to make them suitable for use (by reducing microorganisms to a certain level). Biological decontamination includes measures and procedures of remove or neutralizes pathogens to the extent of eliminating the risk of infection occurrence. It can be categorized as partial biological decontamination and complete biological decontamination.

Partial decontamination is conducted immediately after exposure to the biological agent. Is implemented by an individual or group (self-decontamination, mutual decontamination) and includes the following actions: shaking, brushing and cleaning of clothing and footwear, washing the opening parts of the body with soap and water and rubbing the skin with disinfectants. These procedures should not be applied uncritically. In the case of the dispersion of biological agents in the air, which is most effective and thus the most likely way of usage of biological weapons, shaking and brushing clothes will lead to a re-creation of aerosols, which will increase the risk of infection, rather than reduce it. The use of disinfectants for the decontamination of the skin can also in some cases be counterproductive, or it can speed up creation of infection (as with the skin form of anthrax). In such cases the most effectually decontamination is to limit the washing of the discovered body parts with soap and seep water, than to leave the endangered area (if feasible) and then as soon as possible to conduct a full decontamination.

Full (complete) decontamination is performed outside the zone of biological weapons action. Decontamination of people is implemented in the form of bathing and showering, a thorough washing of hairy parts of the body and nails. Clothing should be carefully previously striped down, avoiding creation of the aerosols, and then packed in plastic bags or other packaging that can be hermetically closed. The packaging should be visibly marked. Next, the items and equipment are subjected to the action of physical and chemical agents, and is most effective when it is possible to be exposed to high temperatures (such as incineration, autoclaving, dry sterilization, boiling).

Depending on the methods used, the decontamination can be conducted by chemical and physical methods. 


\section{Secururity}

Chemical decontamination make biological warfare agents to be safe through the use of disinfectants. In the use of chemicals as a means for biological decontamination, it should be approached carefully. There is not existing chemical that could be applied in practice and to be safe for human health and the environment, and to be an effective tool for biological decontamination. It is recommended that contaminated parts be washed with $0.5 \%$ sodium hypochlorite if available, in contact time of 10 to 15 minutes. The solution can be applied with a cloth or swab or can be sprayed. Just as with hypochlorite in chemical decontamination, this solution should not be put in the eye, in the abdominal cavity or nerve tissue. It will neutralize or do almost harmful all biological agents in approximately 5 minutes. For decontamination of fabric clothing or equipment, it should be used a solution of $5 \%$ hypochlorite. For decontamination of equipment in normal cleaning should be to 30 minutes in time of contact. The use of the hypochlorite solution in this way is corrosive to most metals and damaging to most fabrics, so they should be washed well and metal surfaces to oiled finishing. It is important to remember that washing with soap and water after washing with hypochlorite for decontamination of biological agents should follow the decontamination of chemical agents, if that kind of decontamination is necessary. ${ }^{185}$ This solution evaporates very quickly at high temperatures, so, if prepared in advance, it should be kept in closed containers. The hypochlorite solution should be placed in clearly marked containers, because it's quite difficult to visually distinguish the solution of $0.5 \%$ than $5 \%$.

Physical methods are concerned with making biological agents harmless by physical means such as heating and radiation. To make agents completely safe, it requires dry heat treatment of 2 hours at $160^{\circ} \mathrm{C}$. If used steam at $121^{\circ} \mathrm{C}$ and 1 atmosphere of pressure ( $15 \mathrm{psi})$, the time can be reduced to 20 minutes, depending on the quantity ${ }^{186}$. The part of the sun's ultraviolet radiation that comes to the surface possesses a disinfectant effect, often combined with drying. Ultraviolet radiation is effective but is difficult to standardize the practice for disinfection or decontamination purposes.

\footnotetext{
185 For mixing hypochlorite solution, vials of calcium hypochlorite granules can be found in the kit for chemical decontamination of some armies (like the US Army M291). 0.5\% solution can be made by placing a package of $170 \mathrm{~g}$ of hypochlorite granules in 18.9 liters of water. The solution of $5 \%$ can be made by placing 8 such packages of $170 \mathrm{gr}$ in 18.9 liters ( 5 gallons) of water.

${ }^{186}$ The method is known as autoclaving.
} 
The term effective decontamination almost be equated with the concept of sterilization, which is mostly made with natural methods (exposure to high temperatures) or, rarely, with some chemicals (such as ethylene oxide) which requires equipment and means of protection, because that are extremely toxic compounds. The available means for chemical decontamination in most cases destroy the vegetative form of some bacteria, while their effectiveness against spores, various types of bacteria, fungi, protozoa, viruses and microbial toxins that can be used as a biological weapon is variable and incomplete. Therefore, it is always a chance of residual of more or less the amount (dose) of biologically active material, which can cause disease in humans and animals. The use of "effective" chemical substances to neutralize the biological agents, on the other hand, it could create a false impression in the public about the safety of people in certain area. Therefore, the advantage is of previously mentioned acts of a partial or complete physical decontamination (Birtašević 1989). Water and food in the zone of action of biological agents should not be used, regardless of the implemented decontamination, except in case of lack of other sources of supply.

\section{Conclusion}

Biological factors as causes of human suffering and agony, mass disease and death, have followed human civilization since its beginning until today. Biological agents have supported the war for long time and often have decisive influenced the outcome.

It is claimed that reasonable state actors would never used offensive biological weapons. The arguments are that biological weapons cannot be controlled: Weapons can recover and injure the army in offensive with possible greater effect than the one achieved at the target. Agents such as the smallpox (variola) or other air-transmitted viruses likely to be dispersed and would also infect the surrounding states. However, these views are not always related to bacteria. For example, anthrax can be easily controlled and easily created by a very low cost with available laboratory equipment. Also, using microbiological methods, the bacterium can favorably be amended to be effective only in a limited environment such as the range of the target which is clearly distinguishable from the army in offensive. Moreover, such weapons could be used to 
jam advancing army which would make it being vulnerable to counterattack of the defense forces.

Besides the possibility of using in warfare hostilities, lately is very frequent use of biological agents by terrorist organizations, sects or individuals, defined as biological terrorism. Due to the characteristics of these means that make them suitable for achieving certain objectives (such as cost and ease of manufacture, ease of application, the possibility of combining with other agents ...), many microbes and their toxins are used or studied as a biological weapon. Besides the aerosols, agents can be applied through contaminated food and water or directly on the skin and mucous membranes. In recent times by changing the genetic composition with the methods of molecular genetics, it is possible to create organisms with new features that makes those means being more lethal. Since terrorists today search for new and more destructive and easily accessible, it is increased the danger of eventual use of biological weapons first in period of post Cold War and second time now, in an era of reshaping the world order. It indicates actually how mmuch threats from bioterrorism attack is realistic to a greater extent than in past.

The medical community and the public should be acquainted with the epidemiology and control measures needed to increase the likelihood of a peaceful and reasonable behavior if it comes to outbreaks of disease. In fact, the principles that help physicians to develop strategies against diseases are significant when the medical community address the problem of proliferation of biological weapons. For the medical community is necessery further education in order of quickly identification of threats.

The prevention primarily relies on establishing a global norm against the development of such weapons and second, the good security intelligence. Secondary prevention implies timely detection and rapid treatment of the disease. The medical community plays an important role in secondary prevention by participating in monitoring the disease and by reporting and the first indication of the use of biological weapons. As a supplement, continuous research in improvement of surveillance and search after improved diagnostic capabilities, therapeutic agents and effective response plans will further reinforce secondary preventive measures. Lastly, we should not forget the role of tertiary prevention, which limits the disablement caused by the disease. Due to the imperfection of instruments of primary and secondary prevention, the focus of capabilities of countering the biological weapons is on helping those nations who are 


\section{Securianues}

targets for the use of biological weapons. Here, the medical community must be prepared to face the consequences if the "unthinkable" happened.

From a military perspective, a significant component of the protective preexposure preparedness is immunization. In addition, some vaccines are also affordable opportunity for post exposure prophylaxis against potential threats from biological agents. Such vaccines could be used in respond to terrorist attacks against civilians. Drugs and vaccines are equally important to national security as much as combat platforms!

Because of the real danger of bioterrorism, it is more likely that the problems related to it, will have to be faced and resolve by all, sooner or later. It remains the general lesson that it is better to solve it before!

\section{Literature}

1. Basulto, Dominic. 2015. "Everything you need to know about why CRISPR is such a hot technology." The Washington Post. November 11. Accessed February 28, 2016.

1. https://www.washingtonpost.com/news/innovations/wp/2015/11/04/ everything-you-need-to-know-about-why-crispr-is-such-a-hottechnology/?utm term $=$. a50ceca43b05

2. Birtašević B. 1989. "Biološko oružje i zaštita". In Vojna enciklopedija. Beograd: Vojnoizdavaćki i novinski centar, 387-419.

3. Cieslak J. Theodore, George W. Christopher, Mark G. Kortepeter, John R. Rowe, Julie A. Pavlin, Randall C. Culpepper, and Edward M. Eitzen, Jr. 2000. "Immunization against Potential Biological Warfare Agents". In clinical infectious disease, Vol 30, Issue 6: IDSA, 843-850.

4. Čobeljić M. 2001. „Biološki napad. “ In Opšta epidemiologija, edited by Radovanović Z. and Vlajinac H. Vol IV, Beograd: Nauka.

5. Dennis, Carina.2001. The bugs of the war. In Nature, 411, 232-235

6. Garrett, Laurie. 2003. Betrayal of Trust: The Collapse of Global Public Health. Oxford: Oxford University Press.

7. Geissler Erhard. 1986. Biological and toxin weapons today. SIPRI, Oxford University Press, 55-76. 
8. Judith F., et al., 1999. Document:Bioterrorism Readiness Plan: A template for Healthcare facilities. Department of the Navy USA.

9. Kelle A. 2009. "Security issues related to synthetic biology. " In The technoscience and its societal conse- quences, edited by Schmidt M, Kelle A, Ganguli-Mitra A, de Vriend H. Chapter 7. Berlin: Springer

10. Lockwood, A. Jeffrey. 2008. Six-legged Soldiers: Using Insects as Weapons of War. New York: Oxford University Press. 9-26

11. Navas, Enrique. 2002. "Problems associated with potential massive use of antimicrobial agents as prophylaxis or therapy of a bioterrorist attack." In Clinical Microbiology and Infection. Vol. 8, 534-539.

12. Pirofski L, Casadevall A. 1998. The use of licensed vaccines for active immunization of the immunocompromised host. Clin Microbiol Rev. 11:1-26. Accessed April 28,2016.

https://www.ncbi.nlm.nih.gov/pmc/articles/PMC121373/

13. Richmond J.Y., and R.W. McKinney. 1999. Biosafety in microbiological and biomedical laboratories, $4^{\text {th }}$ ed. Washington: U.S. department of health and human services.

14. Treadwell, Tracee. 2003. "Epidemiological Clues to Bioterrorism". Public Health Reports, 118 March-April, 93-94.

15. Wheelis Mark et al. 2006. Deadly Cultures: Biological Weapons Since 1945. Harvard University Press. 